Modern Physics Letters A

(C) World Scientific Publishing Company

\title{
Recent Progress in Double Beta Decay
}

\author{
Steven R. Elliott \\ Physics Division, Los Alamos National Laboratory, Los Alamos, New Mexico, 87545 USA. \\ elliotts@lanl.gov
}

Received (Day Month Year)

Revised (Day Month Year)

\begin{abstract}
At least one neutrino has a mass of about $50 \mathrm{meV}$ or larger. However, the absolute mass scale for the neutrino remains unknown. Studies of double beta decay offer hope for determining the absolute mass scale. Furthermore, the critical question: Is the neutrino its own antiparticle? is unanswered. In particular, zero-neutrino double beta decay $(0 \nu \beta \beta)$ can address the issues of lepton number conservation, the particle-antiparticle nature of the neutrino, and its mass. A summary of the recent progress in $0 \nu \beta \beta$, and the related technologies will be discussed in the context of the future $0 \nu \beta \beta$ program.
\end{abstract}

Keywords: neutrinoless decay, massive Majorana neutrinos, matrix elements, experimental search for $0 \nu \beta \beta$ decay

PACS Nos.: 11.30.Fs, 14.60.Pq, 23.40.-s

\section{Introduction}

Two-neutrino double beta decay $(2 \nu \beta \beta)$ can be described as 2 neutrons simultaneously beta decaying within a nucleus emitting $2 \beta$ particles and $2 \bar{\nu}$ s. If the neutrino has certain characteristics, the alternative process of zero-neutrino double beta decay $(0 \nu \beta \beta)$ may occur where the neutrino is exchanged as a virtual particle between two neutrons and only $\beta$ particles are emitted in the final state. Understanding neutrino properties motivates the study of this process.

At least one neutrino has a mass of about $50 \mathrm{meV}$ or larger. However, the absolute mass scale for the neutrino remains unknown. Furthermore, the critical question "Is the neutrino its own antiparticle?" is unanswered. Studies of $0 \nu \beta \beta$ can address the issues of lepton number conservation, the particle-antiparticle nature of the neutrino, and the neutrino mass scale. Recent experimental results have demonstrated the increasing reach of the technologies used to search for $0 \nu \beta \beta$. In addition, theoretical progress in understanding the nuclear physics involved has also been impressive. All indications are that upcoming generations of $0 \nu \beta \beta$ experiments will be sensitive to neutrino masses in the exciting range below $50 \mathrm{meV}$.

The half-life of $0 \nu \beta \beta$ is directly related to the neutrino mass. But the half life is very long; at least greater than $10^{25}$ years. Hence any search for the rare peak in 
a spectrum resulting from $0 \nu \beta \beta$ must minimize the background of other processes that may take place in a detector. In addition, deducing a neutrino mass value from a half-life measurement or limit requires an understanding of the transition matrix element, which is technically difficult to calculate.

In this article, a summary of the the related nuclear physics of $\beta \beta$ will be discussed in the context of the future $0 \nu \beta \beta$ program. Numerous reviews have been written on this topic and provide great detail on this exciting science program. (See for example Refs. 1, 2, 3, 4, 5, 6.)

\section{2. $0 \nu \beta \beta$ and Neutrino Mass}

The decay rate for $0 \nu \beta \beta$ can be written:

$$
\left[T_{1 / 2}^{0 \nu}\right]^{-1}=G_{0 \nu}\left|M_{0 \nu}\right|^{2}\left\langle m_{\beta \beta}\right\rangle^{2}
$$

where $T_{1 / 2}^{0 \nu}$ is the half-life of the decay, $G^{0 \nu}$ is the kinematic phase space factor, $M_{0 \nu}$ is the matrix element corresponding to the $0 \nu \beta \beta$ transition, and $\left\langle m_{\beta \beta}\right\rangle$ is the effective Majorana neutrino mass. $G^{0 \nu}$ contains the kinematic information about the final state particles, and is calculable to the precision of the input parameters (though use of different nuclear radius values in the scaling factors of $G^{0 \nu}$ and $M_{0 \nu}$ have previously introduced some confusion-7).

Cosmology is sensitive to the sum of the neutrino mass eigenstates $(\Sigma)$ and $\beta$ decay endpoint measurements determine a different combination $\left(\left\langle m_{\beta}\right\rangle\right)$ of mass eigenvalues and neutrino mixing parameters than $0 \nu \beta \beta$. The three techniques, therefore, provide complementary information on neutrino physics. The three equations are given by:

$$
\begin{aligned}
\left\langle m_{\beta \beta}\right\rangle & =\left|\sum_{j} m_{j} U_{e j}^{2}\right|=\left.\left|m_{1}\right| U_{e 1}\right|^{2}+m_{2}\left|U_{e 2}\right|^{2} e^{i \phi_{1}}+m_{3}\left|U_{e 3}\right|^{2} e^{i \phi_{2}} \mid \\
\left\langle m_{\beta}\right\rangle & =\sqrt{\sum_{j} m_{j}^{2} U_{e j}^{2}} \\
\Sigma & =\sum_{j} m_{j}
\end{aligned}
$$

where $m_{j}$ are the neutrino mass eigenstates and $U_{e j}$ are the neutrino mixing matrix elements. Equation 2 is written for 3 light-mass neutrinos. The CP violating phases denoted by $\phi_{1}$ and $\phi_{2}$ are functions of the mixing matrix phase and the Majorana phases. (See Ref. 2 for example.) Present cosmological constraints on $\Sigma$ are in the range 440-760 meV $\sqrt{89}$ and upcoming measurements hope to reach a sensitivity below $100 \mathrm{meV} 10111$. The KATRIN tritium $\beta$-decay experiment hopes to reach a sensitivity for $\left\langle m_{\beta}\right\rangle$ of $200 \mathrm{meV} \sqrt{12}$. $\beta$-decay neutrino-mass results would have the least model dependency of the 3 techniques. $0 \nu \beta \beta$ results will be the most sensitive laboratory 
measurements of neutrino mass if the neutrino is Majorana. The interpretation of cosmology results would be greatly enhanced by a laboratory neutrino mass result with which to constrain models.

An open question in neutrino physics is whether or not the lightest neutrino mass eigenstate is the dominant component of the electron neutrino. If so, we refer to the neutrino mass spectrum as being normal. If not, we refer to it as inverted. Figure 1 shows the effective Majorana neutrino mass as a function of the lightest neutrino mass for these 2 possibilities using the neutrino oscillation parameters from Ref. [13.

\section{Cancellation Effects of CP Phases}

Figure 1 seems to indicate that a large fraction of the potential parameter space within the normal hierarchy can result in a negligible $\left\langle m_{\beta \beta}\right\rangle$ even if neutrinos are Majorana particles. This is a bit misleading because for the expression in Eqn. 2 to result in a small $\left\langle m_{\beta \beta}\right\rangle$, specific values of the mixing elements, mass eigenstates and phases must conspire to cancel. Barring some symmetry that requires such a cancellation, this would be a unnatural coincidence. In fact the fraction of the parameter space that would result in a cancellation is rather small if the parameter values are random. In Fig. 2 one sees that for a given value of $m_{1}$ in the region of parameter space that can potentially suffer such cancelations, about $5 \%$ of the $\phi_{1}-\phi_{2}$ space results in $\left\langle m_{\beta \beta}\right\rangle$ less than $1 \mathrm{meV}$.
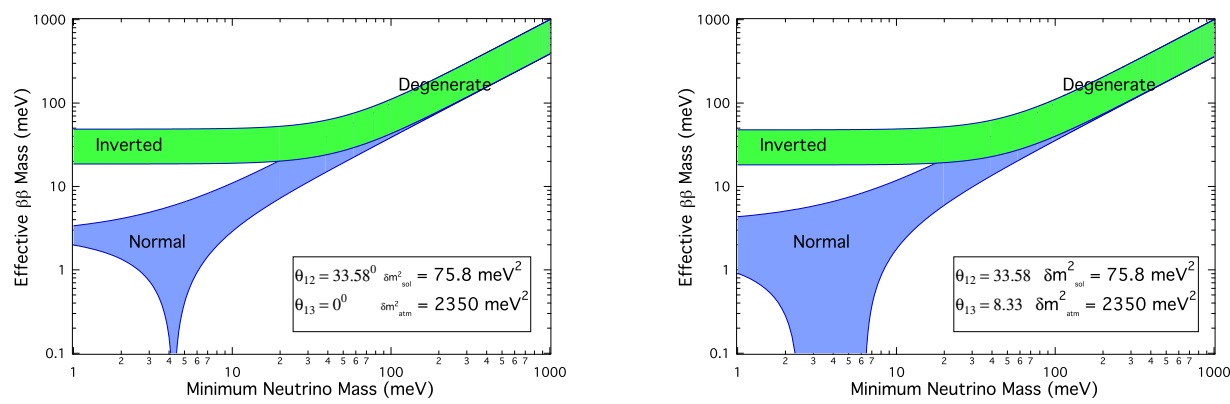

Fig. 1. The effective Majorana neutrino mass as a function of the lightest neutrino mass. In the left panel, $\theta_{13}$ was taken to be zero, whereas in the right panel it is set to the best fit value in recent global fits.

\section{Nuclear Physics and $\beta \beta$}

The observation of $0 \nu \beta \beta$ would have profound qualitative physics conclusions. However, the interpretation of those results quantitatively requires knowledge of $M_{0 \nu}$. Furthermore, an accurate knowledge of $M_{0 \nu}$ has benefits for experimental design. Most nuclear matrix element calculations involve either the quasiparticle random 

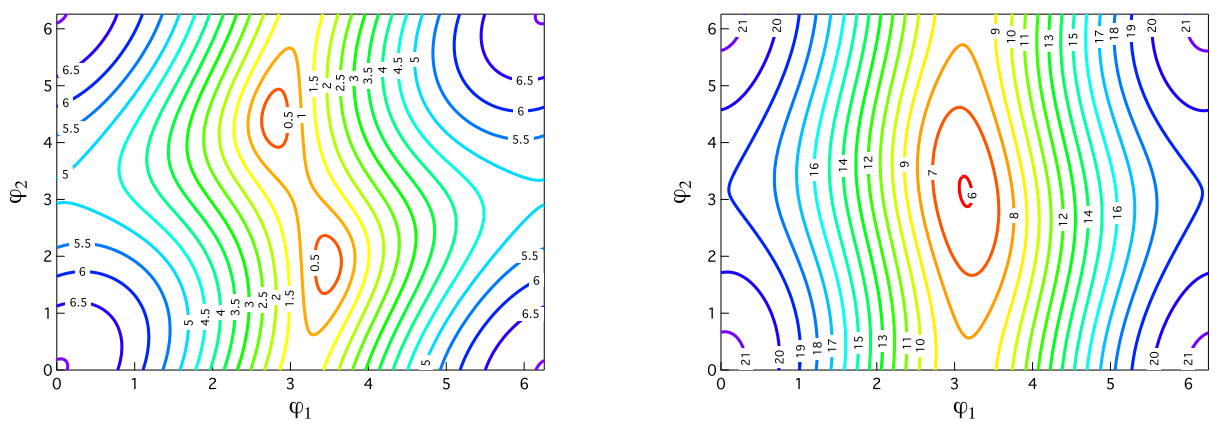

Fig. 2. Contour plots of $\left\langle m_{\beta \beta}\right\rangle$ as a function of the CP violating phases. The mixing angle values were chosen as in recent global analyses. In the left panel $m_{1}$ was chosen to be $4.5 \mathrm{meV}$ as that resides near the center of the normal hierarchy branch of the $\left\langle m_{\beta \beta}\right\rangle$ region that can be subject to significant cancellations for specific parameter values. In the right panel, $m_{1}$ was chosen to be 20 $\mathrm{meV}$, where no cancelation is anticipated.

Table 1. A list of recent $0 \nu \beta \beta$ experiments and their $90 \%$ confidence level (except as noted) limits on $T_{1 / 2}^{0 \nu}$. The $\left\langle m_{\beta \beta}\right\rangle$ limits are those quoted by the authors using the $M_{0 \nu}$ of their choice.

\begin{tabular}{|c|c|c|c|c|}
\hline Isotope & Technique & $T_{1 / 2}^{0 \nu}$ & $\left\langle m_{\beta \beta}\right\rangle(\mathrm{eV})$ & Reference \\
\hline${ }^{48} \mathrm{Ca}$ & $\mathrm{CaF}_{2}$ scint. crystals & $>1.4 \times 10^{22} \mathrm{y}$ & $<7.2-44.7$ & 14 \\
\hline${ }^{76} \mathrm{Ge}$ & enr Ge det. & $>1.9 \times 10^{25} \mathrm{y}$ & $<0.35$ & 15 \\
\hline${ }^{76} \mathrm{Ge}$ & enr $\mathrm{Ge}$ det. & $\left(1.19_{-0.50}^{+2.99}\right) \times 10^{25} \mathrm{y}(3 \sigma)$ & $0.24-0.58$ & 16 \\
\hline${ }^{76} \mathrm{Ge}$ & enr $\mathrm{Ge}$ det. & $>1.57 \times 10^{25} \mathrm{y}$ & $<(0.33-1.35)$ & 17 \\
\hline${ }^{82} \mathrm{Se}$ & Thin metal foils and tracking & $>3.6 \times 10^{23} \mathrm{y}$ & $<(0.89-2.54)$ & 18 \\
\hline${ }^{96} \mathrm{Zr}$ & Thin metal foils and tracking & $>9.2 \times 10^{21} \mathrm{y}$ & $<(7.2-19.5)$ & 19 \\
\hline${ }^{100} \mathrm{Mo}$ & Thin metal foils and tracking & $>1.1 \times 10^{24} \mathrm{y}$ & $<(0.45-0.93)$ & 18 \\
\hline${ }^{116} \mathrm{Cd}$ & ${ }^{116} \mathrm{CdWO}_{4}$ scint. crystals & $>1.7 \times 10^{23} \mathrm{y}$ & $<1.7$ & 20 \\
\hline${ }^{128} \mathrm{Te}$ & geochemical & $>7.7 \times 10^{24} \mathrm{y}$ & $<(1.1-1.5)$ & 21 \\
\hline${ }^{130} \mathrm{Te}$ & $\mathrm{TeO}_{2}$ bolometers & $>2.8 \times 10^{24} \mathrm{y}$ & $<(0.3-0.7)$ & 22 \\
\hline${ }^{136} \mathrm{Xe}$ & Xe disolved in liq. scint. & $>5.7 \times 10^{24} \mathrm{y}$ & $<(0.3-0.6)$ & 23 \\
\hline${ }^{150} \mathrm{Ne}$ & Thin metal foil within TPC & $>1.8 \times 10^{22} \mathrm{y}$ & N.A. & 24 \\
\hline
\end{tabular}

phase approximate (QRPA) technique or the nuclear shell model (NSM). Although the two methods have a similar starting point (a Slater determinant of independent particles), they are complementary in their treatment of correlations. QRPA uses a large number of active nucleons in a large space but with a specific type of correlation suited for collective motion. NSM uses a small number of nucleons within a limited space but with arbitrary correlations. Three additional techniques have recently been used to estimate $M_{0 \nu}$. These include the interacting boson model $(\text { IBM- } 2)^{25}$, the projected Hartree-Fock-Bogolyubov (PHFB) ${ }^{26}$, and the Energy Density Functional (EDF) $)^{27}$ techniques.

Recent publications 28 29|30|31|32|33|34 have elucidated the causes of the historical disparity of results from QRPA calculations of $M_{0 \nu}$. As a result, the technique now provides a uniform result and Ref. 34 summarizes the values for several isotopes. 
The NSM has also seen a resurgence of activity with studies of input micro-physics and its influence on $M_{0 \nu}{ }^{35 \mid 36}$ with recent values given in Ref. [37, This progress indicates that the agreement between the various calculations is improving, but further work is needed to reduce the uncertainty to levels required for comparisons between $0 \nu \beta \beta$ results from different isotopes $38 / 39$.

A previous workshop $\underline{40}$ was dedicated to nuclear physics studies that would support the understanding of $\beta \beta$. Much work has been done since that workshop, not only on ideas presented there but others that have arisen since. Below we focus on the impact of some of these related nuclear physics measurements.

\subsection{Atomic Masses}

One key aspect of $0 \nu \beta \beta$ searches is that the energy of the transition is known because the $\mathrm{Q}$-value is deduced from the nuclear masses. The $\mathrm{Q}$-values for many $\beta \beta$ transitions, however, were previously not well known. In fact in a number of cases, experimental resolution was better than the uncertainty in the endpoint energy. Recent Penning-trap measurements $\underline{4142}$ have been providing a wealth of data to eliminate this uncertainty. In the case of ${ }^{130} \mathrm{Te}$, a new Q-value measurement ${ }^{43}$ resulted in an approximate $5 \% T_{1 / 2}^{0 \nu}$ limit change due to a shift in the endpoint energy at which the experiment searched $22 \sqrt{44}$ indicating the importance of the Q-value. For experiments trying to observe resonant radiative zero-neutrino double electron capture $45[46(0 \nu 2 E C)$, knowledge of the Q-value is critical in order to choose isotopes for which the resonance is possible. A number of these isotope masses have been measured recently also, but no strong resonance has yet been identified. Reference 47 provides a summary of mass measurements applicable to $0 \nu 2 E C$ and lists a number of remaining possible candidate isotopes for which a resonance may exist.

\subsection{Neutron Reactions}

Neutron reactions can result in background for $\beta \beta$ but the cross sections are often unavailable and require measurement in order to predict the impact on an experiment. For example, the ${ }^{207} \mathrm{~Pb}\left(\mathrm{n}, \mathrm{n}^{\prime} \gamma\right)$ reaction can produce a $3062-\mathrm{keV} \gamma$ ray $\underline{48}$. This is a dangerous background for $0 \nu \beta \beta$ using ${ }^{76} \mathrm{Ge}$ because the double escape peak energy coincides with the Q-Value. Many of the cross sections for such reactions, including this example ${ }^{\sqrt{49}}$ were not well known and measurements are required. Reactions such as ${ }^{76} \mathrm{Ge}(\mathrm{n}, 9 \mathrm{n}){ }^{68} \mathrm{Ge}$ are also dangerous ${ }^{50}$ as they result from cosmic-ray induced neutrons while materials reside on the Earth's surface. The cross sections for such large $\Delta A$ transitions are small however, and many are also frequently not yet measured.

\subsection{Transfer Reactions and Pair Correlation Studies}

Proton- and neutron-removing transfer reactions have played a critical role in providing tests of the nuclear structure used for the calculation of $M_{0 \nu}$. The difference 
in the nucleon configuration of the initial and final nuclei is an important input to $M_{0 \nu}$. Reactions such as $(\mathrm{d}, \mathrm{p}),(\mathrm{p}, \mathrm{d}),\left(\alpha,{ }^{3} \mathrm{He}\right)$, and $\left({ }^{3} \mathrm{He}, \alpha\right)$ were used to study the occupation of valence neutron orbits in ${ }^{76} \mathrm{Ge}$ and ${ }^{76} \mathrm{Se} \mathrm{e}^{51}$. For protons, $\left(\mathrm{d},{ }^{3} \mathrm{He}\right)$ reactions were studied on these isotopes 52 . The correlation of pairs of neutrons was measured in this system using the $(\mathrm{p}, \mathrm{t})$ reaction 53 . These data were then used to

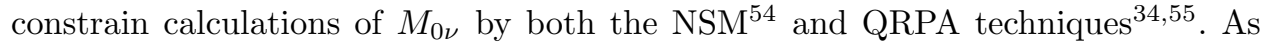
a result, the matrix element calculations of both techniques shifted such that the difference between them was reduced. Studies on other $\beta \beta$ isotopes are underway.

\subsection{Precise $2 \nu \beta \beta$ Data}

Accurate half-lives for $2 \nu \beta \beta^{28}$ along with $\beta^{ \pm}$and electron capture decay data of the intermediate nuclei30 can help determine the $g_{p p}$ parameter used in QRPA calculations. Being able to calculate $2 \nu \beta \beta$ rates can be also considered a necessary, if not sufficient condition for calculating $0 \nu \beta \beta$ rates. Reference [56] summarizes $2 \nu \beta \beta$ measurements and compares to the $M_{2 \nu}$ deduced. In addition the recent $2 \nu \beta \beta$ measurements of ${ }^{136} \mathrm{Xe}{ }^{57 / 23}$ will impact future analyses of $0 \nu \beta \beta$.

\subsection{Other Nuclear Physics Measurements for the Study of $M_{0 \nu}$}

A variety of other proposals for studying the physics related to $M_{0 \nu}$ have been made and we summarize them here.

Charge exchange reactions on parent \& daughter: Charge exchange reactions, such as $(\mathrm{p}, \mathrm{n}),(\mathrm{n}, \mathrm{p}),\left({ }^{3} \mathrm{He}, \mathrm{t}\right)$, etc., can provide data on the Gammov-Teller transition strengths of interest for $\beta \beta^{58}$. The Fermi part of the total $\beta \beta$-decay nuclear matrix element might be studied in charge-exchange reactions 5 .

Muon capture: The $\beta \beta$ virtual transition proceeds through levels of the intermediate nucleus. For $2 \nu \beta \beta$ intermediate $1^{+}$states are involved, whereas for $0 \nu \beta \beta$ all $\mathrm{J}^{+}$states participate. Theoretical calculation of the relative strengths of these virtual states is very difficult. Muon capture on the final nucleus 60 also excites all multipoles and therefore provides additional experimental data with which to compare calculation techniques.

Neutrino Cross Sections: Matrix element studies could be done with neutrino beams ${ }^{61}$. By varying the average neutrino beam energy, specific multipoles of the intermediate nucleus can be excited. The strengths of both legs of the $\beta \beta$ transition could be studied by using both $\overline{\nu_{e}}$ and $\nu_{e}$.

Electromagnetic Transitions to Isobaric Analogue States: The matrix element for the $\beta$ decay of an intermediate nucleus can be measured by observing the $(\gamma, \mathrm{p})$ through isobaric-analog-state excitation on the daughter nucleus ${ }^{62}$.

\section{Upcoming Experiments}

Within the next 3-5 years, a number of new experiments will begin to provide data on $0 \nu \beta \beta$. All of these programs have a chance to test a recent claim of the observation of $0 \nu \beta \beta^{63}$. The validity of this claim has been debated ${ }^{64[65] 66 / 67 / 68}$ and 
therefore confirmation is required. Table 2 summarizes these upcoming experiments along with various longer term efforts. These projects are complementary in that some emphasize isotope mass (EXO-200, KamLAND-Zen, SNO+), others energy resolution (CUORE, GERDA, MAJORANA), and still others include precision tracking (NEXT, SuperNEMO). All can potentially lead to technologies with enough isotope mass to reach sensitivity to the inverted hierarchy neutrino mass scale. It is anticipated that the sensitivity of these projects will result in mass limits of 100 $\mathrm{meV}$ or less by about mid decade.

Here we summarize a number of the experiments, however, there are a great number of other $\mathrm{R} \& \mathrm{D}$ projects that are too numerous to discuss in detail here, but the key points of each are included in Table 2.

\subsection{CUORE}

CUORE (Cryogenic Underground Observatory for Rare Events) ${ }^{69}$, and its planned prototype CUORE- 06 , use $\mathrm{TeO}_{2}$ crystals operated as bolometers. This program builds on the previously successful CUORICINO experiment ${ }^{44}$. The project exploits the high natural abundance $(33.8 \%)$ of ${ }^{130} \mathrm{Te}$ as the $\beta \beta$ isotope. The planned use of 988 crystals provides a total of $203 \mathrm{~kg}$ of ${ }^{130} \mathrm{Te}$. These bolometers operate at low temperature $(\approx 8-10 \mathrm{mK})$ where the heat capacity is very small. Therefore an energy deposit due to $\beta \beta$ can be measured as a temperature rise in the detector. Energy resolution of $5 \mathrm{keV}$ at the $2528-\mathrm{keV}$ endpoint has been achieved. CUORE-0 and CUORE are being assembled at Gran Sasso and CUORE-0 expects to start data taking early 2012 with the first tower of crystals $\left(10 \mathrm{~kg}{ }^{130} \mathrm{Te}\right)$.

\section{2. $E X O-200$}

EXO-200 (Enriched Xenon Observatory) is operating at the Waste Isolation Pilot Plant (WIPP). The experiment consists of $200 \mathrm{~kg}$ of Xe enriched to $80.6 \%$ in ${ }^{136} \mathrm{Xe}$ housed in a liquid time projection chamber. This detector measures energy through both ionization and scintillation and is capable of effectively rejecting $\gamma$ rays through topological cuts. Encouragingly, EXO-200 has recently claimed the first observation of $2 \nu \beta \beta$ in ${ }^{136} \mathrm{Xe} \mathrm{e}^{57}$. Initial results on $0 \nu \beta \beta$ should be forthcoming. The project is also a prototype for a planned 1-10 tonne sized experiment ${ }^{70}$ that may include the ability to identify the $\beta \beta$ daughter ${ }^{136} \mathrm{Ba}$ in real time, effectively eliminating all classes of background except that due to $2 \nu \beta \beta$.

\subsection{GERDA}

GERDA (GERmanium Detector Array) started physics data taking November 2011 at Gran Sasso ${ }^{71}$. This project uses high purity Ge detectors, which provide the best energy resolution of any double beta decay experiment. In their Phase-I configuration, they immersed the enriched detectors used by the IGEX ${ }^{17]}$ and HeidelbergMoscow ${ }^{72}$ collaborations bare into a large volume of liquid argon. The liquid argon 
vessel is itself enclosed within a large water tank. The Ge detectors total $17.66 \mathrm{~kg}$ of $\mathrm{Ge}$ metal which is enriched to $86 \%$ in ${ }^{76} \mathrm{Ge}$. Initial results should be available soon. For their Phase-II configuration, they have obtained additional enriched material and plan to add an additional $\approx 20 \mathrm{~kg}$ of p-type detectors with small read out electrode $\frac{73 / 74 / 75}{7}$ and begin their commissioning in late 2012 or early 2013.

\subsection{KamLAND-Zen}

KamLAND-Zen ${ }^{23}$ (Kamioka Liquid Anti-Neutrino Detector, ZEro Neutrino double beta decay) is an extension of the KamLAND ${ }^{76}$ experiment. KamLAND is a 6.5$\mathrm{m}$ radius balloon filled with $1000 \mathrm{t}$ of liquid scintillator, submerged inside a 9-m radius stainless-steel sphere filled with $3000 \mathrm{t}$ of mineral oil with PMTs mounted on the wall. The cavity outside this sphere is filled with water also instrumented with PMTs. KamLAND was built to search for reactor anti-neutrinos and the extension is intended as a search for $0 \nu \beta \beta$. The collaboration added an additional low-background miniballoon into the inner sphere that contains $13 \mathrm{t}$ of liquid scintillator loaded with $330 \mathrm{~kg}$ of dissolved Xe gas enriched to $91 \%$ in ${ }^{136} \mathrm{Xe}$. This detector at the Kamioka mine in Japan began $\beta \beta$ operation in September 2011 and initial results include an improved limit on $T_{1 / 2}^{0 \nu}$ for ${ }^{136} \mathrm{Xe}$ and a measurement of $T_{1 / 2}^{2 \nu}$ that agrees with the recent EXO-200 result.

\subsection{MAJORANA}

The Majorana Demonstrator ${ }^{77 / 78 / 79}$ is being constructed as a prototype to show that building a large mass Ge detector with low-enough background to study the inverted hierarchy neutrino-mass scale is feasible. The project consists of $40 \mathrm{~kg}$ of Ge p-type point-contact detectors $\$ 80 \mid 77$ enclosed within 2 separate electroformed copper cryostats. Up to $30 \mathrm{~kg}$ of these detectors will be fabricated from Ge enriched to $86 \%$ in ${ }^{76} \mathrm{Ge}$. The cryostats will be contained within a shield that consists of electroformed $\mathrm{Cu}$, OFHC $\mathrm{Cu}$, lead, cosmic ray veto system, and plastic. Electroformed $\mathrm{Cu}$ is used due to its extreme purity ${ }^{78 \mid 81}$. The experiment is being constructed at the Sanford Underground Research Facility (SURF) at the old Homestake gold mine in Lead, SD. The first cryostat is planned for commissioning in 2013.

The MAJORANA and GERDA collaborations are cooperating in efforts to design a large-mass Ge detector. The configuration of such an experiment will be optimized based on the outcomes of the Demonstrator and GERDA Phase-II.

\subsection{NEXT}

NEXT $82[83$ (Neutrino Experiment with Xenon TPC) intends to use $>100 \mathrm{~kg}$ of Xe enriched to $\sim 90 \%$ in ${ }^{136} \mathrm{Xe}$. The detector will be a moderate-density gas TPC $\sim 0.08$ $\mathrm{g} / \mathrm{cm}^{3}$ that will also detect scintillation light. By operating at low pressures $(\approx 15$ bar), the design should not only provide good energy resolution, but also permit tracking that allows fairly detailed track reconstruction to confirm that candidate 
events involve two electrons moving in opposite directions. The collaboration has recently demonstrated impressive 1\% FWHM resolution in a limited fiducial volume device. Construction is scheduled to start in 2012 with commissioning to start in 2014. It will operate at the Laboratorio Subterráneo de Canfranc in Spain (LSC).

\section{7. $\mathrm{SNO}+$}

$\mathrm{SNO}+{ }^{84 \mid 85}$ is an outgrowth of the SNO (Sudbury Neutrino Observatory) experiment that observed solar neutrinos ${ }^{86}$. For $\mathrm{SNO}+$, the heavy water within the large acrylic vessel is replaced by scintillator with dissolved Nd. For a fractional loading of $0.1 \%$ by weight, they will use $43.6 \mathrm{~kg}$ of ${ }^{150} \mathrm{Nd}$, although higher loadings are being studied. In addition to $\beta \beta$ the experiment can also study pep and CNO solar neutrinos along with reactor and geo anti-neutrinos. The experiment is being constructed at SNOLAB in Canada and is expected to begin data taking in mid 2013 with scintillator and with added Nd soon thereafter.

\subsection{SuperNEMO}

The SuperNEMO 87 proposal builds on the great success of the NEMO-3 (Neutrino Ettore Majorana Observatory) experiment, which measured $2 \nu \beta \beta$ rates in 7

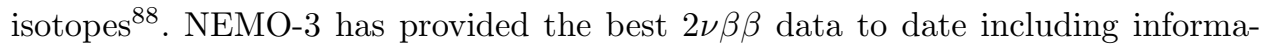
tion on single-electron energy distributions and opening angles. The design uses calorimetry to measure energies and tracking to gather kinematical information about the individual electrons. SuperNEMO will improve on NEMO-3 by using a larger mass of isotope, lowering backgrounds, and improving the energy resolution. The present design is for $100 \mathrm{~kg}$ of ${ }^{82} \mathrm{Se}$, but other isotopes are being considered. It will have a modular design of 20 thin-source planes of $40 \mathrm{mg} / \mathrm{cm}^{2}$ thickness. Each source will be contained within a geiger-mode drift chamber enclosed by scintillator and phototubes. Timing measurements from digitization of the scintillator and drift chamber signals will provide topological information such as the event vertex and particle directionality. The modules will be surrounded by water and passive shielding. A one-module demonstrator with $7 \mathrm{~kg}$ of ${ }^{82} \mathrm{Se}$ is planned to be in operation by 2014. This Demonstrator will have only passive shielding. The complete experiment will be ready by the end of the decade in an extension of the LSM Modane in the Frejus Tunnel in France.

\section{The Number of Required Experimental Results}

Although the existence of $0 \nu \beta \beta$ would prove that neutrinos are massive Majorana particles $\frac{100}{}$, it is not clear which lepton violating process might actually mediate the decay. Since we now know that light massive neutrinos do exist and would mediate $0 \nu \beta \beta$ if they are Majorana particles, that is the simplest model that could incorporate $0 \nu \beta \beta$. It is this hypothesis that is expressed in Eqn. 1 Nevertheless, other physics might be present including: heavy Majorana neutrino exchange, right- 
Table 2. A summary list of the $0 \nu \beta \beta$ proposals and experiments.

\begin{tabular}{|c|c|c|c|c|c|}
\hline Experiment & Isotope & Mass & Technique & Present Status & Location \\
\hline AMoRE 8990 & ${ }^{100} \mathrm{Mo}$ & $50 \mathrm{~kg}$ & $\mathrm{CaMoO}_{4}$ scint. bolometer crystals & Development & Yangyang \\
\hline CANDLES 91 & ${ }^{48} \mathrm{Ca}$ & $0.35 \mathrm{~kg}$ & $\mathrm{CaF}_{2}$ scint. crystals & Prototype & Kamioka \\
\hline CARVEL 92 & ${ }^{48} \mathrm{Ca}$ & 1 ton & $\mathrm{CaF}_{2}$ scint. crystals & Development & Solotvina \\
\hline $\mathrm{COBRA}^{93}$ & ${ }^{116} \mathrm{Cd}$ & $183 \mathrm{~kg}$ & enr Cd CZT semicond. det. & Prototype & Gran Sasso \\
\hline CUORE-c 69 & ${ }^{130} \mathrm{Te}$ & $11 \mathrm{~kg}$ & $\mathrm{TeO}_{2}$ bolometers & Construction - 2012 & Gran Sasso \\
\hline CUORE 69 & ${ }^{130} \mathrm{Te}$ & $203 \mathrm{~kg}$ & $\mathrm{TeO}_{2}$ bolometers & Construction - 2013 & Gran Sasso \\
\hline $\mathrm{DCBA}^{94}$ & ${ }^{150} \mathrm{Ne}$ & $20 \mathrm{~kg}$ & ${ }^{e n r} \mathrm{Nd}$ foils and tracking & Development & Kamioka \\
\hline EXO-200 & ${ }^{136} \mathrm{Xe}$ & $160 \mathrm{~kg}$ & Liq. ${ }^{e n r} \mathrm{Xe} \mathrm{TPC} /$ scint. & Operating - 2011 & WIPP \\
\hline EXd70 & ${ }^{136} \mathrm{Xe}$ & $1-10 \mathrm{t}$ & Liq. enr Xe TPC/scint. & Proposal & SURF \\
\hline GERDA $^{71}$ & ${ }^{76} \mathrm{Ge}$ & $\approx 35 \mathrm{~kg}$ & enr Ge semicond. det. & Operating - 2011 & Gran Sasso \\
\hline GSC 95 & ${ }^{160} \mathrm{Gd}$ & 2 ton & $\mathrm{Gd}_{2} \mathrm{SiO}_{5}$ : Ce crys. scint. in liq. scint. & Development & \\
\hline KamLAND-Zer 96 & ${ }^{136} \mathrm{Xe}$ & $400 \mathrm{~kg}$ & enr Xe disolved in liq. scint. & Operating - 2011 & Kamioka \\
\hline LUCIFER $97 / 98$ & ${ }^{82} \mathrm{Se}$ & $18 \mathrm{~kg}$ & ZnSe scint. bolometer crystals & Development & Gran Sasso \\
\hline MAJORANA $\sqrt{77 / 78 / 79}$ & ${ }^{76} \mathrm{Ge}$ & $26 \mathrm{~kg}$ & enr Ge semicond. det. & Construction - 2013 & SURF \\
\hline MOON 99 & ${ }^{100} \mathrm{Mo}$ & $1 \mathrm{t}$ & enr Mofoils/scint. & Development & \\
\hline SuperNEMO-Dem 87 & ${ }^{82} \mathrm{Se}$ & $7 \mathrm{~kg}$ & $e^{e n r}$ Se foils/tracking & Construction - 2014 & Fréjus \\
\hline SuperNEMC 87 & ${ }^{82} \mathrm{Se}$ & $100 \mathrm{~kg}$ & ${ }^{e n r}$ Se foils/tracking & Proposal - 2019 & Fréjus \\
\hline NEXT 8283 & ${ }^{136} \mathrm{Xe}$ & $100 \mathrm{~kg}$ & gas TPC & Development - 2014 & Canfranc \\
\hline $\mathrm{SNO}+8485$ & ${ }^{150} \mathrm{Nd}$ & $55 \mathrm{~kg}$ & Nd loaded liq. scint. & Construction - 2013 & SNOLab \\
\hline
\end{tabular}

handed currents (RHC), and exchange mechanisms that arise from R-Parity violating supersymmetry (RPV SUSY) models.

In contrast to Eqn. 1, the $0 \nu \beta \beta$ rate can be written more generally:

$$
\left[T_{1 / 2}^{0 \nu}\right]^{-1}=G^{0 \nu}\left|M_{0 \nu} \eta\right|^{2}
$$

where $\eta$ is a general lepton number violating parameter (LNVP) that was previously given by $\left\langle m_{\beta \beta}\right\rangle$. The LNVP $\eta$ contains all of the information about lepton number violation.

The LNVP takes on different forms for different $0 \nu \beta \beta$ mechanisms. In addition, $M_{0 \nu}$ may also depend on the mechanism. The heavy-particle models represent a large class of theories that involve the exchange of high-mass $(>1 \mathrm{TeV})$ particles. For example, leptoquarks ${ }^{101}$ have very similar $M_{0 \nu}$ to RPV SUSY ${ }^{102}$. Left-right symmetric models can lead to right-handed current models $\frac{103}{}$ or heavy neutrino exchange models 101 . Scalar bilinears ${ }^{104}$ might also mediate the decay but explicit matrix elements have not been calculated yet. For SUSY and left-right symmetric models, effective field theory ${ }^{105}$ has been used to determine the form of the effective hadronic operators from the symmetries of the $0 \nu \beta \beta$-decay operators in a given theory. In all of these cases the estimates of $M_{0 \nu}$ are not as advanced as that for light neutrino exchange and more work is needed.

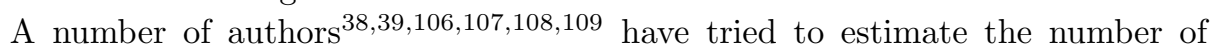
measurements required to discern the underlying physics. The assumption that is critical to these arguments is that the spread in $M_{0 \nu}$ due to different models reflects the true variation, which is clearly speculative given the uncertainties still 
remaining in those calculations. With this assumption in mind, the conclusion is that 3 or more experiments with a precision of about $20 \%$ for both experiment and theory are needed to have any hope of discerning the underlying physics. There are other motivations that require multiple $0 \nu \beta \beta$ experimental results 110 . The need to prove that the observation is indeed $0 \nu \beta \beta$ and not an unidentified background is not the least among them. Therefore, a general conclusion from these papers, and those similar to them, indicate that at least 3 (and very likely 4) $0 \nu \beta \beta$ experiments along with significant theoretical effort are warranted. The need for and utility of several precision experimental results is the critical conclusion.

\section{Toward the Normal Hierarchy Neutrino-Mass Scale}

Atmospheric neutrino oscillation results indicate that at least one neutrino mass eigenstate is about $50 \mathrm{meV}$ or more. In the inverted hierarchy with a small value for the lightest $m_{i}\left(m_{\text {lightest }}\right),\left\langle m_{\beta \beta}\right\rangle$ would be about $20-50 \mathrm{meV}$. At this value for $\left\langle m_{\beta \beta}\right\rangle$ the $0 \nu \beta \beta$ half life would be near $10^{27} \mathrm{y}$. It is this scenario that motivates the size of the tonne-scale experiments that will evolve from the projects listed in Section 5 In the normal hierarchy, when $m_{\text {lightest }}$ is near $0,\left\langle m_{\beta \beta}\right\rangle$ is $5-10 \mathrm{meV}$. To reach such sensitivity, $0 \nu \beta \beta$ experiments must be capable of observing a half-life near $10^{29} \mathrm{y}$. This will require 100 tonnes of isotope. The technology for enriching isotopes at this quantity is not yet cost effective and research will be required to develop it. Furthermore, energy resolution will be critical to make sure that background from the tail of the $2 \nu \beta \beta$ spectrum doesn't mask the $0 \nu \beta \beta$ signal due to its much faster decay rate. Techniques to fabricate a very large number of high-resolution crystal detectors in a cost effective way must be developed or, alternatively, improving the resolution of large scintillator detectors is required. At any mass scale, once $0 \nu \beta \beta$ is observed, technologies that can measure the full kinematic information of the process will be a critical component of the $\beta \beta$ program.

The various $\beta \beta$ experiments prior to 2010 used up to $\sim 10 \mathrm{~kg}$ of isotope and were successful in measuring $2 \nu \beta \beta$ in $\sim 10$ isotopes and demonstrated sensitivity to a half-life for $0 \nu \beta \beta$ near $10^{25} \mathrm{y}$. In the upcoming years until 2015 , experiments using up to a few hundred $\mathrm{kg}$ will built and operated as discussed in Section 5 From these experiments we anticipate a robust test of the recent claim and either limits or measurements near $100 \mathrm{meV}$ or better. At that point in time, there will be a decision point depending on whether $0 \nu \beta \beta$ is convincingly detected or not. If it is observed, the program will proceed toward a large number of experiments sensitive to the appropriate $\left\langle m_{\beta \beta}\right\rangle$ scale in an effort to elucidate the underlying physics as discussed in Section 6. If not, then the program will push on to the tonne-scale and atmospheric mass-scale sensitivity below $50 \mathrm{meV}$ followed by another decision point. Depending on whether $0 \nu \beta \beta$ is observed or not, a collection of experiments will be developed the better to study the decay or the program will aspire to even greater sensitivity - reaching toward the normal hierarchy mass scale.

Reference 111 relates a cautionary tale about double beta decay experiments. 
It describes a number of past $\beta \beta$ efforts that produced, in some cases, incorrect conclusions, and in others, enticing indications of new physics that took time to understand as background or instrumental effects. Although the article paints some past experimental efforts with a overly negative broad brush, it does make clear that as we reach for ever greater sensitivity, we are likely to encounter new background effects with which we are presently unfamiliar. Any claim for $0 \nu \beta \beta$ will take time to confirm and fully understand.

\section{Conclusions}

The worldwide research program in $\beta \beta$ is making fast progress due to the great interest in the subject. This interest arises because we now know that neutrinos have mass and because $0 \nu \beta \beta$ is the only practical way to discover if the neutrino is its own anti-particle. In fact, $0 \nu \beta \beta$ can only exist if neutrinos are massive Majorana particles. Experimental technologies have now advanced to the point that sensitivity to the inverted hierarchy mass scale will soon be achieved. It is also now possible to imagine reaching the normal hierarchy scale even if the concepts aren't yet feasible. However, to interpret a measured decay rate (or limit) in terms of constraints on neutrino parameters requires an understanding of the matrix elements. $\beta \beta$ is a second order weak process that proceeds through many possible intermediate states and therefore the matrix element calculation is a difficult theoretical challenge. Not only has the theory made great recent strides in developing techniques to calculate $M_{0 \nu}$, a number of experimental efforts are investigating the nuclear physics involved to help better understand these calculations. All told, this is a very exciting time for the study of $\beta \beta$.

\section{Acknowledgments}

I gratefully acknowledge the support of the U.S. Department of Energy through the LANL/LDRD Program for this work. I also gratefully acknowledge the support of the U.S. Department of Energy, Office of Nuclear Physics under Contract No. 2011LANLE9BW. I wish to thank Hamish Robertson and Frank Avignone for a careful reading of this manuscript and many useful suggestions. I thank many of my experimental colleagues who read the sections on their respective experiments and improved the descriptions. These include F. T. Avignone III, M. Chen, Yu. Efremenko, G. Gratta, K. Lang, D. Nygren and S. Schonert.

\section{References}

1. Elliott S R and Vogel P 2002 Ann. Rev. Nucl. Part. Sci. 52115

2. Elliott S R and Engel J 2004 J. Phys. G: Nucl. Part. Phys. 30 R 183

3. Avignone F T III, Elliott S R and Engel J 2008 Rev. Mod. Phys. 80481 (Preprint arXiv:0708.1033)

4. Rodejohann W 2011 Int. J. Mod. Phys. E 201833 (Preprint arXiv:1106.1334)

5. Barabash A S 2011 Phys. Part. Nucl. 42613 (Preprint arXiv:1107.5663) 
6. Gómez-Cadenas J J, Martín-Albo J, Mezzetto M, Monrabal F and Sorel M 2011 (Preprint arXiv:1109.5515)

7. Cowell S 2006 Phys. Rev. C 73028501

8. Hannestad S, Mirizzi A, Raffelt G G and Wong Y Y Y 2010 JCAP 08001 (Preprint arXiv:1004.0695)

9. Gonzalez-Garcia M C, Maltoni M and Salvado J 2010 J. High. Ener. Phys. 1008 117 (Preprint arXiv: 1006.3795)

10. Hannestad S, Tu H and Wong Y Y Y 2006 JCAP 0606025 (Preprint astro-ph/ 0603019)

11. Lesgourgues J, Perotto L, Pastor S and Piat M 2006 Phys. Rev. D 73045021 (Preprint astro-ph/0511735)

12. Robertson R G H (KATRIN) 2008 J. Phys. Conf. Ser. 120052028 (Preprint arXiv: 0712.3893)

13. Fogli G L, Lisi E, Marrone A, Palazzo A and Rotunno A M 2011 to appear in Phys. Rev. D (Preprint arXiv:1106.6028)

14. Ogawa I et al. 2004 Nucl. Phys. A $\mathbf{7 3 0} 215$

15. Klapdor-Kleingrothaus H V et al. 2001 Eur. Phys. J. A 12147

16. Klapdor-Kleingrothaus H V, Deitz A, Krivosheina I V and Chkvorez O 2004 Phys. Lett. B $\mathbf{5 8 6} 198$

17. Aalseth C E et al. 2002 Phys. Rev. D. 65092007

18. Barabash A S et al. 2011 Phys. At. Nucl. 74312 (Preprint arXiv:1002.2862)

19. Argyriades J et al. 2010 Nucl. Phys. A 847168 (Preprint arXiv:0906.2694)

20. Danevich F A et al. 2003 Phys. Rev. C 68035501

21. Bernatowicz T et al. 1993 Phys. Rev. C 47806

22. Arnaboldi C et al. 2008 Phys. Rev. C $\mathbf{7 8} 035502$

23. Gando A et al. 2012 (Preprint arXiv:1201.4664

24. Argyriades J et al. 2009 Phys. Rev. C 80032501

25. Barea J and Iachello F 2009 Phys. Rev. C 79044301

26. Chandra R, Chaturvedi K, Rath P, Raina1 P and Hirsch J 2009 Europhys. Lett. 86 32001

27. Rodríguez T R and Martínez-Pinedo G 2010 Phys. Rev. Lett. 105252503 (Preprint arXiv:1008.5260v2)

28. Rodin V A, Faessler A, Šimkovic F and Vogel P 2003 Phys. Rev. C 68044302

29. Rodin V A, Faessler A, Šimkovic F and Vogel P 2006 Nucl. Phys. A 766107 erratum arXiv:0706.4304

30. Suhonen J 2005 Phys. Lett. B 60787

31. Kortelainen M, Civitarese O, Suhonen J and Toivanen J 2007 Phys. Lett. B 647128 (Preprint nucl-th/0701052)

32. Faessler A, Fogli G, Lisi E, Rodin V, Rotunno A and Šimkovic F 2008 Phys. Rev. D 79053001 (Preprint arXiv:0810.5733)

33. Šimkovic F, Faessler A and Vogel P 2009 Phys. Rev. C 79015502 (Preprint arXiv: 0812.0348 )

34. Simkovic F, Faessler A, Müther H, Rodin V and Stauf M 2009 Phys. Rev. C 79 055501 (Preprint arXiv:0902.0331)

35. Caurier E, Nowacki F and Poves A 2008 Eur. Phys. J. A 36195

36. Caurier E, Menendez J, Nowacki F and Poves A 2008 Phys. Rev. Lett. 100052503

37. Menéndez J, Poves A, Caurier E and Nowacki F 2009 Nucl. Phys. A 818139

38. Gehman V M and Elliott S R 2007 J. Phys. G 34667

39. Deppisch F and Päs H 2007 Phys. Rev. Lett. 98232501 (Preprint hep-ph/0612165)

40. Zuber K 2005 summary of the Workshop on: Nuclear matrix elements for neutrinoless 
double beta decay (Durham UK) (Preprint nucl-ex/0511009)

41. Rahaman S et al. 2008 Phys. Lett. B 662111 (Preprint arXiv:0712.3337)

42. Redshaw M et al. 2007 Phys. Rev. Lett. 98053003

43. Redshaw M, Mount B J, Myers E G and Avignone F T III 2009 Phys. Rev. Lett. 102212502

44. Andreotti E et al. 2011 Astropart. Phys. 34822 (Preprint arXiv:1012.3266)

45. Sujkowski Z and Wycech S 2004 Phys. Rev. C 70052501 (Preprint hep-ph/0312040)

46. Wycech S and Sujkowski Z 2004 Acta. Phys. Polon. B 351223

47. Eliseev S 2011 presentation at MEDEX 2011, Prague, Czech Republic (Preprint http://medex11.utef.cvut.cz/talks/Eliseev.pdf)

48. Mei D M, Elliott S R, Hime A, Gehman V and Kazkaz K 2008 Phys. Rev. C 77 054614 (Preprint arXiv:0704.0306)

49. Guiseppe V E, Devlin M, Elliott S R, Fotiades N, Hime A, Mei D M, Nelson R and Perepelitsa D 2009 Phys. Rev. C 79054604 (Preprint arXiv:0809.5074)

50. Elliott S, Guiseppe V, LaRoque B, Johnson R and Mashnik S 2010 Phys. Rev. C 82 054610

51. Schiffer J P et al. 2008 Phys. Rev. Lett. 100112501

52. Kay B P et al. 2009 Phys. Rev. C 79 021301(R)

53. Freeman S J et al. 2007 Phys. Rev. C $\mathbf{7 5} 051301$

54. Menéndez J, Poves A, Caurier E and Nowacki F 2009 Phys. Rev. C 80048501

55. Suhonen J and Civitarese O 2008 Phys. Lett. B 668277

56. Barabash A S 2010 Phys. Rev. C 81035501 (Preprint arXiv:1003.1005)

57. Ackerman N et al. 2011 (Preprint arXiv:1108.4193)

58. Ejiri H 2000 Phys. Rep. 338265

59. Rodin V and Faessler A 2009 Phys. Rev. C 80041302

60. Kortelainen M and Suhonen J 2002 Europhys. Lett. 58666

61. Volpe C 2005 J. Phys. G 31903

62. Ejiri H 2006 Czech. J. Phys. 56459

63. Klapdor-Kleingrothaus H V and Krivosheina I V 2006 Mod. Phys. Lett. A 211547

64. Aalseth C E et al. 2002 Mod. Phys. Lett. A 171475

65. Feruglio F, Strumia A and Vissani F 2002 Nucl. Phys. B 637345

66. Harney H L 2001 (Preprint hep-ph/0205293)

67. Zdesenko Yu G, Danevich F A and Tretyak V I 2002 Phys. Lett. B 546206

68. Kirpichnikov I 2010 (Preprint arXiv:1006.2025)

69. Alessandria F et al. 2011 submitted to Astropart. Phys. (Preprint arXiv:1109. 0494v2)

70. Danilov M et al. 2000 Phys. Lett. B 48012

71. Schönert S et al. 2005 Nucl. Phys. Proc. Suppl. 145242

72. Baudis L et al. 1999 Phys. Rev. Lett. 8341

73. Budjáš D, Heider M B, Chkvorets O, Khanbekov N and Schönert S 2009 JINST 4 P10007

74. Barnabe-Heider M et al. 2010 JINST 5 P10007

75. Agostini M et al. 2011 JINST 6 P03005 (Preprint arXiv:1012.4300)

76. Abe S et al. 2008 Phys. Rev. Lett. 100221803

77. Aguayo E et al. 2011 presented at the American Physical Society Division of Particles and Fields 2011, Providence RI, USA, August 2011 (Preprint arXiv:1109.6913)

78. Phillips D G II et al. 2011 presentation for the Rutherford Centennial Conference on Nuclear Physics, Manchester UK, August 2011 (Preprint arXiv:1111.5578)

79. Schubert A G et al. 2011 submitted to AIP Conference Proceedings, 19th Particles and Nuclei International Conference (PANIC 2011), Massachusetts Institute of 
Technology, Cambridge, MA, USA, July 24-29, 2011 (Preprint arXiv:1109.1567)

80. Barbeau P S, Collar J I and Tench O 2007 JCAP 09009

81. Hoppe E W et al. 2008 J. Radioanal. Nucl. Chem. 277103

82. Gómez E et al. 2011 (Preprint arXiv:1106.3630)

83. Yahlali N et al. 2010 Nucl. Instrum. Meth. Phys. A 617520

84. Chen M C 2005 Nucl. Phys. Proc. Suppl. 14565

85. Chen M C et al. 2008 eConf C080730 (Preprint hep-ex/0810.3694)

86. Ahmad Q R et al. (SNO) 2001 Phys. Rev. Lett. 87071301

87. Arnold R et al. 2010 Eur. Phys. J. C 70927 (Preprint arXiv:1005.1241)

88. Bongrand M et al. 2011 to be published in Proceedings 22nd Rencontres de Blois, July, 2010 (Preprint arXiv:1105.2435)

89. Kim S K 2011 presentation at MEDEX 2011, Prague, Czech Republic (Preprint http://medex11.utef.cvut.cz/talks/Kim.pdf)

90. Lee S J et al. 2011 Astropart. Phys. 34732

91. Kishimoto T 2009 Int. J. Mod. Phys. E 182129

92. Zdesenko Yu G et al. 2005 Astropart. Phys. 23249

93. Zuber K 2001 Phys. Lett. B 5191

94. Ishihara N et al. 2000 Nucl. Instrum. Meth. A 443101

95. Danevich F A 2001 Nucl. Phys. A 694375

96. Efremenko Yu 2011 presentation at MEDEX 2011, Prague, Czech Republic (Preprint http://medex11.utef.cvut.cz/talks/Efremenko.pdf)

97. Giuliani A 2010 presentation at Beyond 2010, Cape Town, South Africa, February 2010

98. Arnaboldi C et al. 2011 Astropart. Phys. 34344 (Preprint arXiv:1006.2721)

99. Ejiri H 2007 Mod. Phys. Lett. A 221277

100. Schechter J and Valle J W F 1982 Phys. Rev. D 252951

101. Hirsch M, Klapdor-Kleingrothaus H, and Kovalenko S 1996 Phys. Lett. B 372181

102. Hirsch M, Klapdor-Kleingrothaus H, and Kovalenko S 1996 Phys. Lett. B 3747

103. Doi M, Kotani T and Takasugi E 1985 Prog. Theor. Phys. 831

104. Klapdor-Kleingrothaus H V and Sarkar U 2003 Phys. Lett. B 55445

105. Prézeau G, Ramsey-Musolf M and Vogel P 2003 Phys. Rev. C 68034016

106. Fogli G L, Lisi E and Rotunno A M 2009 Phys. Rev. D 80015024

107. Šimkovic F, Vergados J and Faessler A 2010 Phys. Rev. D 82113015

108. Faessler A, Fogli G L, Lisi E, Rotunno A M and Šimkovic F 2011 Phys. Rev. D 83 113015

109. Ali A, Borisov A V and Zhuridov D V 2010 Phys. Atom. Nucl. 732083

110. Elliott S R 2006 invited talk at Neutrino 2006 (Preprint nucl-ex/0609024)

111. Tretyak V I 2011 (Preprint arXiv:1112.4183) 